\title{
Relationship between Luminosity, Irradiance and Temperature of star on the orbital parameters of exoplanets
}

\author{
Pintr Pavel ${ }^{1, a}$ \\ ${ }^{1}$ Regional Centre for Special Optics and Optoel. Systems - TOPTEC, Sobotecká 1660, Turnov 51101, Czech Republic
}

\begin{abstract}
For 759 exoplanets detected by radial velocities method we found that distances of exoplanets from central star comply in general Schmidt law and these distances depend on the stellar surface temperature. Every stellar spectral class has a little different distribution. The Luminosity and the Irradiance has not effect on the distribution of distances of exoplanets. We have found the new formulas for calculation of effective temperature of exoplanets for spectral classes F, G, and K. These new formulas we can use for future calculation of habitable planets.
\end{abstract}

\section{Introduction}

Planets are extremely faint objects compared to their parent stars. At visibile wavelenghts, they usually have less than a millionth of their parent star's brithtness. It is very difficult to detect these faint light sources and it is necessary to block the light from the parent star in order to reduce the glare. Detection of light from planet is great challange [1]. Most confirmed extrasolar planets have been found with ground-based telescopes but now many exoplanets have been found also with space-based telescopes. COROT [2] and KEPLER [3], [4] are the two currently active space missions dedicated to searching for extrasolar planets. We know many methods, how to detect the exoplanets. We will use extrasolar planets detected by these methods in our work:

- Radial velocity method,

- Transit method,

- Astrometry method.

The radial velocity method or Doppler method is most productive method of discovering exoplanets [5]. Variations in the star's radial velocity can be detected from displacements in the star's spectral lines due to the Doppler effect. According [6] we cannot determine a planet's true mass only we can set a lower limit of mass.

The transit method has been the second most productive method for the detection of exoplanets. If a planet transits in front of its parent star's disk, then the observed brightness of the star drops by a small amount. The amount depends on its size and on the size of the planet. This method reveals the radius of a planet.

The astrometry method is not well productive method today, because we need precisely measuring a star's position in the sky and observing the changes in that position over time.

The best idea is to confirm exoplanets with a combination of these methods together. A total of 759 planets in 609 extrasolar systems have been identified as of February 11, 2012 [7]. We know also 99 multi-planet extrasolar systems with more than 2 planets in one system [8], [9] and

a e-mail: AstroPintr@seznam.cz
[10]. In [11] it is argued that the physical quantities of the exoplanet are functions of astrophysical parameters of the central (host) stars, such as the stellar luminosity $L$, stellar irradiance $J$ or stellar effective temperature $T_{\text {eff }}$. These main physical parameters we will use in our study.

\section{Definition of parameters and regression analyse}

We have calculated the luminosity of a star according [12]

$$
L=4 \pi R_{\text {Sun }}^{2} \sigma T_{\text {eff }}^{4},
$$

where $R_{\text {Sun }}$ is the stellar radius in radii of the Sun, $\sigma$ is the Stefan-Boltzmann constant.

We have used for stellar irradiance the Stephan-Boltzmann law in form

$$
J=\sigma T_{\text {eff }}^{4} .
$$

We have used for the statistical study of detected extrasolar systems the regression methods. A regression model relates $Y$ to a function of $X$ and $\beta$ in the form

$$
Y=f(X, \beta),
$$

where $\beta$ are unknown parameters, $X$ are independent variables, and $Y$ is a dependent variable. The observed data $\left(x_{i}, y_{i}\right), i=1,2, \ldots, n$, with $n$ the number of observations, satisfy equations

$$
y_{i}=f\left(x_{i}, \beta\right)+\epsilon_{i},
$$

where $\epsilon_{i}$ is an error term.

For a linear regression, we get two parameters $\beta_{0}$ and $\beta_{1}$ and it is valid that

$$
y_{i}=\beta_{0}+\beta_{1} x_{i}+\epsilon_{i} .
$$

The residual

$$
\epsilon_{i}=y_{i}-f_{i}
$$

This is an Open Access article distributed under the terms of the Creative Commons Attribution License 2.0, which permits unrestricted use, distribution, and reproduction in any medium, provided the original work is properly cited.

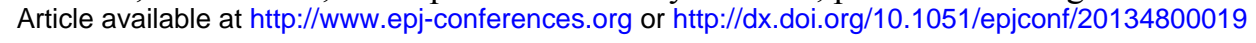


is the difference between the true value of the dependent variable $y_{i}$ and the value of the dependent variable predicted by the model $f_{i}$. We measure the goodness of fit by the coefficient of determination $R^{2}$,

$$
R^{2}=\frac{S S_{\text {reg }}}{S S_{\text {tot }}}
$$

where $S S_{\text {reg }}$ is the regression sum of squares,

$$
S S_{\text {reg }}=\sum_{i=1}^{n}\left(f_{i}-y_{\text {avg }}\right)^{2},
$$

and $S S_{\text {tot }}$ is the total sum of squares,

$$
S S_{\text {tot }}=\sum_{i=1}^{n}\left(y_{i}-y_{\mathrm{avg}}\right)^{2} \text {. }
$$

In (8) and (9), $y_{\mathrm{avg}}$ is the mean of observed values $y_{i}$. When the model function is not linear in the parameters, the sum $\sum_{i=1}^{n} \epsilon_{i}^{2}$ must be minimized by an iterative procedure.

In our recent work [13] we have studied the dependence of orbital parameters of exoplanets. We have studied the distance of planets $r_{\mathrm{p}}$ from the central star on the parameter $v_{\mathrm{p}} r_{\mathrm{p}}$, where the parameter $v_{\mathrm{p}}$ is orbital velocity of planets. In this work we will study the parameters $r_{\mathrm{p}} T_{\text {eff }}$, $r_{\mathrm{p}} L$ and $r_{\mathrm{p}} J$ on the orbital parameter $v_{\mathrm{p}} r_{\mathrm{p}}$ for the stellar spectral classes.

\section{Results for stellar spectral classes}

The spectral class of a star is a designated class of a star describing the ionization of its photosphere, giving an objective measure of the photosphere's temperature. Most stars are currently classified using letters $\mathrm{O}, \mathrm{B}, \mathrm{A}, \mathrm{F}, \mathrm{G}, \mathrm{K}$ and $\mathrm{M}$, where $\mathrm{O}$ stars are the hottest and $\mathrm{M}$ stars are the coolest. We have indentified more than 80 exoplanets for the statistical study only in these spectral classes:

- F class with $T_{\text {eff }}$ between $6000-7500 \mathrm{~K}$,

- G class with $T_{\text {eff }}$ between $5200-6000 \mathrm{~K}$,

- K class with $T_{\text {eff }}$ between $3700-5200 \mathrm{~K}$.

We can find for stars of the spectral type $\mathrm{F}$ from figure 1 that the parameter $r_{\mathrm{p}} T_{\text {eff }}$ depends on the parameter $v_{\mathrm{p}} r_{\mathrm{p}}$ with the coefficient of determination $R^{2}=0.997$. The predicted equation of regression is in the form

$$
r_{\mathrm{p}} T_{\mathrm{eff}} \approx K x^{1.9963},
$$

where $K=4 \times 10^{-17}$ in unit $\left[s K m^{-1}\right]$ and $x=v_{\mathrm{p}} r_{\mathrm{p}}$.

It is valid for the stellar spectral type $\mathrm{F}$ that the parameter $r_{\mathrm{p}} L$ has big scattering with the coefficient of determination $R^{2}=0.923$. We get better results for the parameter $r_{\mathrm{p}} J$ with the value of reliability $R^{2}=0.995$. It is obvious from figure 1 that the parameter $r_{\mathrm{p}} T_{\text {eff }}$ affects mainly the orbits of exoplanets for the stellar spectral type $\mathrm{F}$.

We can find for stars belonging to the spectral type $\mathrm{G}$ from figure 2 that the parameter $r_{\mathrm{p}} T_{\text {eff }}$ depends on the parameter $v_{\mathrm{p}} r_{\mathrm{p}}$ with the coefficient of determination $R^{2}=$ 0.995. The obtained equation of regression is in the form

$$
r_{\mathrm{p}} T_{\mathrm{eff}} \approx K x^{1.976}
$$
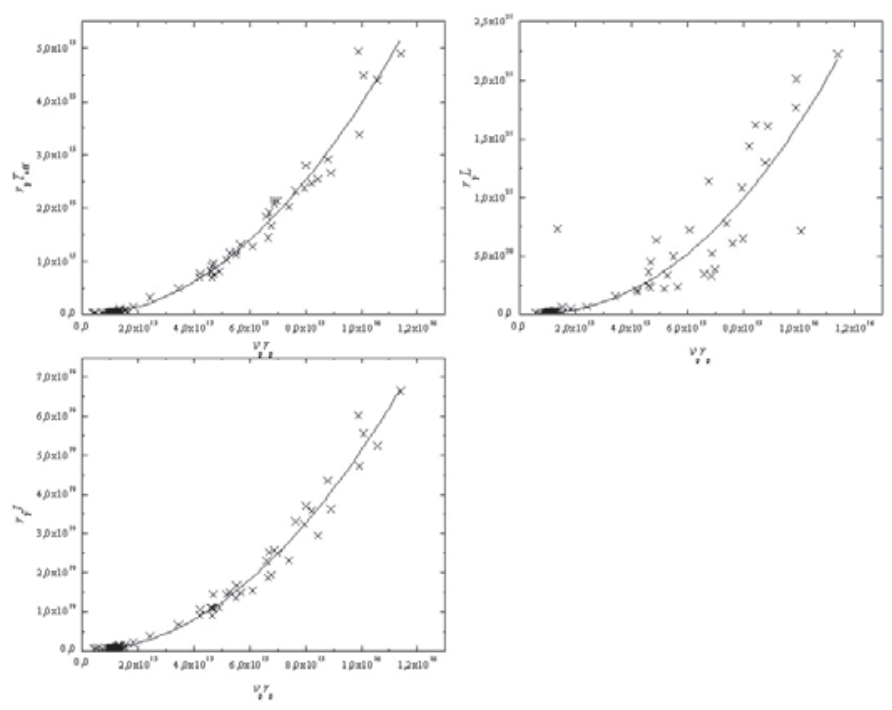

Fig. 1. Dependence of the parameters $r_{\mathrm{p}} T_{\mathrm{eff}}, r_{\mathrm{p}} L$, and $r_{\mathrm{p}} J$ on $v_{\mathrm{p}} r_{\mathrm{p}}$ for the stellar spectral class F. According to the regression analysis, the best is the power interpolation with the coefficient of determination $R^{2}=0.997$ for the parameter $r_{\mathrm{p}} T_{\text {eff }}$.
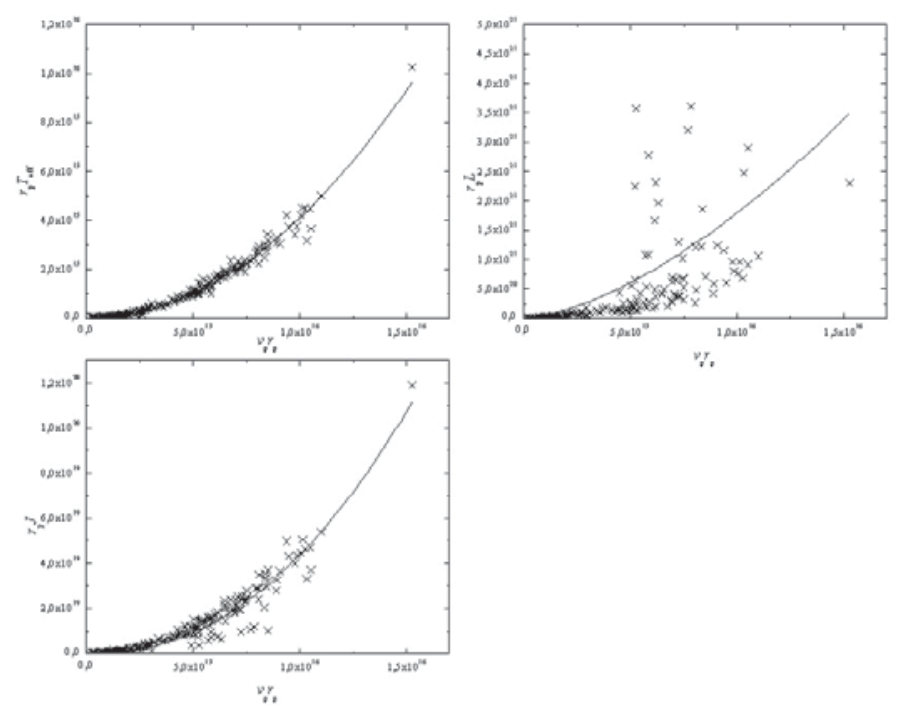

Fig. 2. Dependence of the parameters $r_{\mathrm{p}} T_{\mathrm{eff}}, r_{\mathrm{p}} L$, and $r_{\mathrm{p}} J$ on the parameter $v_{\mathrm{p}} r_{\mathrm{p}}$ for the stellar spectral class G. According to the regression analysis, the best is the power interpolation with the coefficient of determination $R^{2}=0.983$ for the parameter $r_{\mathrm{p}} T_{\text {eff }}$.

where $K=1 \times 10^{-16}$ in unit $\left[s K m^{-1}\right]$ and $x=v_{\mathrm{p}} r_{\mathrm{p}}$.

We observe for the stellar spectral type $G$ that the parameter $r_{\mathrm{p}} L$ has big scattering with the coefficient of determination $R^{2}=0.895$. We get better results for the parameter $r_{\mathrm{p}} J$ with the coefficient of determination $R^{2}=0.979$. From this it follows that the parameter $r_{\mathrm{p}} T_{\text {eff }}$ affects mainly the orbits of exoplanets. 

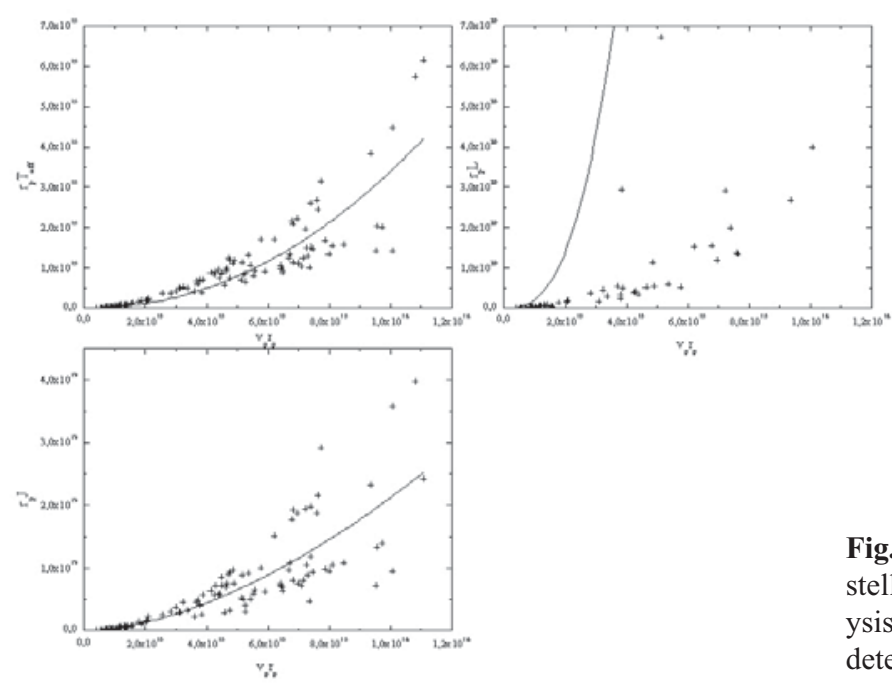

Fig. 3. Dependence of the parameters $r_{\mathrm{p}} T_{\mathrm{eff}}, r_{\mathrm{p}} L$, and $r_{\mathrm{p}} J$ on $v_{\mathrm{p}} r_{\mathrm{p}}$ for the stellar spectral class $\mathrm{K}$. According to the regression analysis, the best is the power interpolation with the coefficient of determination $R^{2}=0.776$ for the parameter $r_{\mathrm{p}} T_{\text {eff }}$.

We can find for stars of the spectral type $\mathrm{K}$ from figure 3 that the parameter $r_{\mathrm{p}} T_{\text {eff }}$ depends on the parameter $v_{\mathrm{p}} r_{\mathrm{p}}$ with the coefficient of determination $R^{2}=0.776$. The predicted equation of regression is in the form

$$
r_{\mathrm{p}} T_{\mathrm{eff}} \approx K x^{1.807}
$$

where $K=4 \times 10^{-14}$ in unit $\left[s K m^{-1}\right]$ and $x=v_{\mathrm{p}} r_{\mathrm{p}}$.

It is valid for stars of the stellar spectral type $\mathrm{K}$ that the parameters $r_{\mathrm{p}} L$ and $r_{\mathrm{p}} T_{\text {eff }}$ have the same regressions with the coefficient of determination $R^{2}=0.974$. This is the main difference from stars of the spectral type $G$. The parameter $r_{\mathrm{p}} T_{\text {eff }}$ affects mainly the orbits of exoplanets for stars of the spectral type K. We can see two types of distributions in figure 3. Many exoplanets are described by the power regression, but some exoplanets deviate from this function.

\section{Temperature of exoplanets}

We can calculate the effective temperature of the exoplanets $T_{\text {eq }}$ by equating the energy received from the star and the energy radiated by the exoplanet, under the black-body approximation in the form

$$
T_{\mathrm{eq}}^{4}=\frac{R_{\mathrm{Star}}^{2} T_{\mathrm{eff}}^{4}}{4 r_{\mathrm{p}}^{2}},
$$

where $R_{\text {Star }}$ is radius of star. In [14] they have calculated $T_{\text {eq }}$ for Kepler exoplanet candidates according the formula

$$
T_{\text {eq }}=T_{\text {eff }} \sqrt{\frac{R_{\text {Star }}}{2 r_{\mathrm{p}}}}\left[f\left(1-A_{B}\right)\right]^{1 / 4},
$$

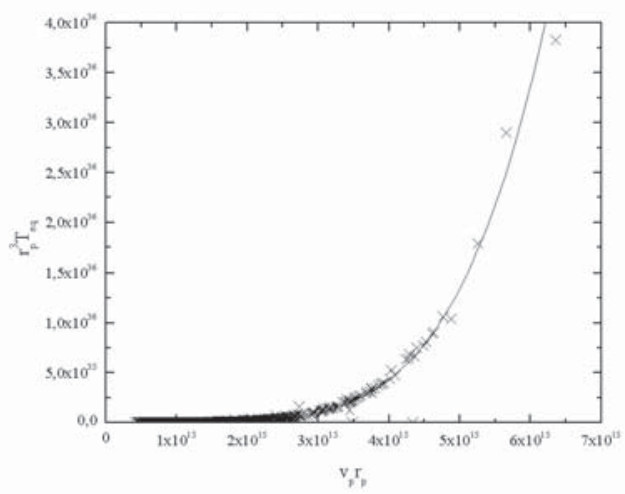

Fig. 4. Dependence of the parameters $r_{\mathrm{p}}^{3} T_{\mathrm{eq}}$ on the $v_{\mathrm{p}} r_{\mathrm{p}}$ for the stellar spectral type $\mathrm{F}$ and $\mathrm{G}$. According to the regression analysis, the best is the power interpolation with the coefficient of determination $R^{2}=0.9987$ for the parameter $r_{\mathrm{p}}^{3} T_{\mathrm{eq}}$.

where $f=1$ is factor of full atmospheric thermal circulation and $A_{B}$ is the Bond albedo. In [14] they have assumed $A_{B}=0.3$.

We have applied our regression methods on the 2321 Kepler exoplanet candidates for the calculation of the parameter $T_{\mathrm{eq}}$. We have found according the regression methods the dependence of parameters $r_{\mathrm{p}}^{3} T_{\text {eq }}$ on the $v_{\mathrm{p}} r_{\mathrm{p}}$.

The obtained equation is from figure 4 with the coefficient of regression $R^{2}=0.9987$ for spectral class $\mathrm{F}$ and $\mathrm{G}$ in the form

$$
y=C x^{5},
$$

where $C=5 \times 10^{-43}$ in unit $[s K m]$ or

$$
r_{\mathrm{p}}^{3} T_{\mathrm{eq}}=C\left(v_{\mathrm{p}} r_{\mathrm{p}}\right)^{5}
$$

and after the modification we can get new formula in the form

$$
T_{\mathrm{eq}}=C v_{\mathrm{p}}^{5} r_{\mathrm{p}}^{2} .
$$

The obtained equation from figure 5 with the coefficient of regression $R^{2}=0.9688$ for spectral class $\mathrm{K}$ is in the form

$$
y=C x^{4.52}
$$

where $C=1 \times 10^{-35}$ in unit $[\mathrm{sKm}]$ and after modification we get the new formula

$$
T_{\text {eq }}=C v_{\mathrm{p}}^{4.52} r_{\mathrm{p}}^{1.52}
$$

We can use these new formulas for determination of habitable planets [15] and [16] in the future. Habitable zone is the region around a star within which it is theoretically possible for a planet with sufficient atmospheric pressure to maintain liquid water on its surface. The habitable planets should have surface temperature $T_{\text {eq }}$ between $273 \mathrm{~K}-373 \mathrm{~K}$. But it is very important to calculate also the green house effect of exoplanet atmosphere and also the factor $f$ of thermal circulation. 


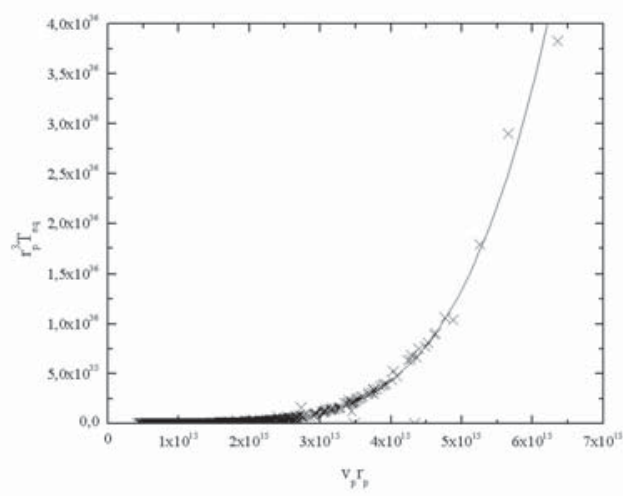

Fig. 5. Dependence of the parameters $r_{\mathrm{p}}^{3} T_{\text {eq }}$ on the $v_{\mathrm{p}} r_{\mathrm{p}}$ for the stellar spectral type $\mathrm{K}$. According to the regression analysis, the best is the power interpolation with the coefficient of determination $R^{2}=0.9688$ for the parameter $r_{\mathrm{p}}^{3} T_{\mathrm{eq}}$.

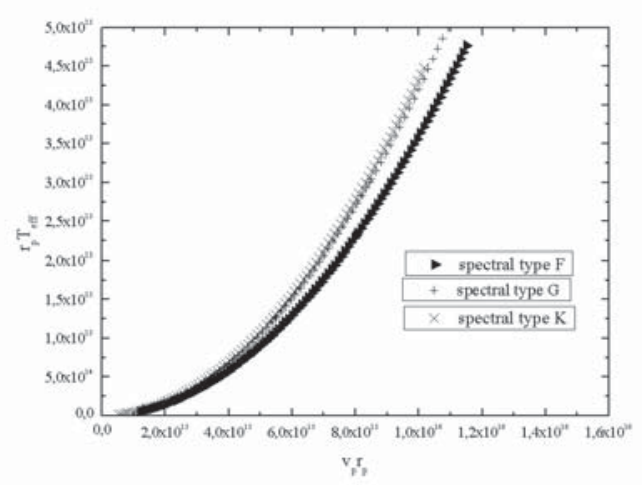

Fig. 6. Dependence of the parameter $r_{\mathrm{p}} T_{\text {eff }}$ on $r_{\mathrm{p}} v_{\mathrm{p}}$ for different stellar spectral classes.

\section{Conclusion}

We have found for 759 exoplanets detected by the method of radial velocities that the distances of exoplanets from the central star obey, in general, the Schmidt law and these distances $r_{\mathrm{p}}$ depend on the stellar surface temperature $T_{\text {eff }}$. Each stellar spectral class has a little different regression of $r_{\mathrm{p}} T_{\text {eff }}$ on the $r_{\mathrm{p}} v_{\mathrm{p}}$. The parameter $r_{\mathrm{p}} L$ has big scattering than the parameter $r_{\mathrm{p}} J$. From this it follows that the parameter $r_{\mathrm{p}} T_{\text {eff }}$ affects mainly the distribution of exoplanets (see figure 6).

We have found for the 2321 candidates from the Kepler mission new formulas (17) and (19) for the calculation of the effective temperature $T_{\text {eq }}$ of exoplanets for the spectral classes $F, G$ and $\mathrm{K}$. We can use these formulas for future calculation of habitable planets.

\section{Acknowledgements}

This work was supported by the European Regional Development Fund and the Ministry of Education, Youth and Sports of the Czech Republic in the Project No. CZ.1.05/2.1.
00/03.0079: Research Center for Special Optics and Optoelectronic Systems (TOPTEC).

\section{References}

1. Perryman M.: The Exoplanet Handbook, 149, (2011).

2. Deleuil M., Moutou C., Borde P, et al.: arXiv:1105.1887v1, (2011).

3. Borucki, J. W., et al.: Science 327, 977, (2010).

4. Borucki, J. W., et al.: The Astrophysical Journal 728, 117, (2011).

5. Pepe F., Lovis C., Segransan D., Benz W., Bouchy F., Dumusque X., Mayor M., Queloz D., Santos N. C., Udry S.: Astronomy and Astrophysics 534, (2011).

6. Rodler F., Lopez-Morales M., Ribas I.: The Astrophysical Journal Letters 753,1, (2012).

7. Schneider, J.: http://exoplanet.eu/catalog.php.

8. Fressin, F., et al.: Nature 482, 195, (2011).

9. Marcy, G. W., et al.: The Astrophysical Journal 581, 1375, (2002).

10. Marcy, G. W., et al.: The Astrophysical Journal 556, 296, (2001).

11. Braun, K. V.: European Planetary Science Congress Abstracts, 6, (2011).

12. Luminosity of stars. Australian Telescope Outreach and Education, Available from: http://outreach.atnf.csiro.au/education/senior/ astrophysics /photometry/luminosity.html (2004).

13. Smarandache, F., Christianto, V., Pintr, P.: Quantization and Discretization at Large Scales, 15-26, (2012).

14. Batalha, N. M., et al.: Available from: http://arxiv: astro-ph/1202.5852/, (2012).

15. Morris E.T.: Astronomical Circumstances, Available from: http://www.planetarybiology.com.

16. Jones B.W., Sleep N. P., Underwood D. R.: The Astrophysical Journal 649 2, (2006). 